\title{
Combining Query Translation with Query Answering for Efficient Keyword Search
}

\author{
Günter Ladwig and Thanh Tran \\ Institute AIFB, Karlsruhe Institute of Technology, Germany \\ \{guenter.ladwig, duchthanh.tran\}@kit.edu
}

\begin{abstract}
Keyword search has been regarded as an intuitive paradigm for searching not only documents but also data, especially when the users are not familiar with the data and the query language. Two types of approaches can be distinguished. Answers to keywords can be computed by searching for matching subgraphs directly in the data. The alternative to this is keyword translation, which is based on searching the data schema for matching join graphs, which are then translated to queries. Answering these queries is performed in the later stage. While clear advantages have been shown for the approaches based on query translation, we observe that processing done during query translation has some overlaps with the processing needed for query answering. We propose a tight integration of query translation with query answering. Instead of using the schema, we employ a bisimulation-based structure index graph. Searching this index for matching subgraphs results not only in queries, but also candidate answers. We propose a set of algorithms which allow for an incremental process, where intermediate results computed during query translation can be reused for query answering. In experiments, we show that this integrated approach consistently outperforms the state of the art.
\end{abstract}

\section{Introduction}

Graph structured data has attracted much attention recently, which is partly due to the massive availability of RDF. There are billions of freely available RDF triples, hosted by data providers involved in the LOD project. This number is increasing, just like the amount of RDFa data associated with Web pages.

In this paper, we present an approach for keyword search on graph structured data. Keyword search has been regarded as an intuitive paradigm for exploring and searching for data, especially in the case where the users are not familiar with the data and the query language. Approaches for computing structured answers from keywords exist for different data models, including relational, XML and graph structured data such as RDF [2/312|910|1411].

State of the Art. Two different types of approaches can be distinguished. With direct keyword query answering, answers to keywords are computed by searching for matching subgraphs directly in the data, i.e. subgraphs which connect the data elements matching the keywords 3129 . Implementing such an approach 
amounts to building a native engine for keyword search. Specific indexes and storage mechanisms have to be provided.

Alternatively, keyword search can be supported by computing translations in the form of queries, which can then be processed using an existing database engine. We refer to this as keyword query translation. Examples of database extensions for keyword search include DBXplorer [2] and Discover [11. These systems are based on finding candidate networks, basically join expressions constructed using information found in the schema. These candidate networks are used to instantiate a number of SQL queries. Similarly, [18, extracts a schema from the RDF data graph. During online processing, this schema is augmented with elements matching the user keywords. Keyword translation is performed by finding matching subgraphs in this augmented schema representing possible queries. Processing the queries derived from them is finally performed using the underlying RDF store.

Keyword search based on translation has several advantages [18. The produced queries can be presented to the user, thereby facilitating comprehension of the results and in particular, enabling query refinement. Moreover, keyword search can be performed efficiently: keyword translation is relatively fast, as exploration for subgraphs is performed on the schema. Since this is much smaller than the actual data, exploration for queries is much faster than exploration for answers in the data graph. For query answering, optimization capabilities of the database engine can be leveraged. For keyword search on RDF, it has been shown in [18] that combining fast query translation with state of the art query answering is faster than direct keyword query answering [3129].

While clear advantages have been shown for translation-based approaches, we observe that processing done during query translation has overlaps with processing needed for query answering. We propose a tight integration of query translation with query answering. The main contributions of our approach are:

- We propose a novel process which tightly combines query translation with query answering. This is based on the observation that for query translation, keywords are matched against the data. Then, possible join graphs between these keyword matching elements are obtained. These operations are similar to data retrieval and join operations performed during query answering.

- Instead of a schema, we employ a bisimulation-based structure index. Searching this index for join graphs results not only in queries, but also candidate answers. This allows for an incremental process, where intermediate results computed during query translation can be reused for query answering.

- For efficient and incremental processing, we elaborate on new algorithms for all step in this process. We propose the decomposition keyword queries into segments such that search using these segments result in entities that can be further processed during query answering. The algorithm proposed for join graph search leverages existing strategies for exploration, but computes both join graphs and candidate answers. A special query answering algorithm is proposed for processing join graphs, which takes candidate answers as inputs. 
In experiments, we show that this integrated and incremental process for keyword search consistently outperforms the state of the art.

Outline. We start with a problem definition in Section 2. A brief overview of our approach is presented in Section 3. In Sections 4, 5 and 6, we describe the major steps keyword mapping, query translation and answering. Evaluation results are provided in Section 8. We conclude with a summary in Section 9.

\section{Problem}

In this section, we present models for the data and queries. Then, we define the problem that we elaborate on.

Data Model. We deal with general graph structured data defined as follows:

Definition 1. A data graph is an $R D F$ graph $G=\left(V=V_{E} \uplus V_{D}, L=\right.$ $\left.L_{R} \uplus L_{A}, E\right)$ where $V_{E}$ are entities (RDF resources), $V_{D}$ are data values ( $R D F$ literals), $L_{R}$ and $L_{A}$ are drawn from the set of object properties (relations) and data properties (attributes) labels, and E represent edge instances.

Query Model. Two different types of queries are distinguished in this setting: (1) the system query $q_{s}$, which is the one finally used by the query engine to retrieve answers and (2) the user query $q_{u}$, which is the one actually entered by the user. System queries $q_{s}$ are conjunctive queries of the following type:

Definition 2. A conjunctive query $q$ is an expression of the form $p_{1} \wedge \ldots \wedge p_{n}$, where $p_{n} \in P$ are query atoms of the form $p\left(n_{1}, n_{2}\right)$ with $n_{1}$, $n_{2}$ being variables or constants otherwise, and $p_{n}$ are called predicates. We distinguish between relation query atoms $p_{r} \in L_{R}$ and attribute query atoms $p_{a} \in L_{A}$.

Regarded as the basic block for querying graph structured data (RDF), these queries have been the focus of recent work on RDF query processing [11915].

The user query $q_{u}$ is a keyword query, i.e. $q_{u}=\left\{k_{1}, \ldots, k_{n}\right\}$ where each $k_{i}$ is a keyword. Users enter keyword queries because they are not familiar with the formal query language, the schema or the data. Fig. 11 shows a data graph and example queries.

Conjunctive Query Answering. Fig. 1k illustrates that since variables can interact in an arbitrary way, $q_{s}$ is essentially a graph pattern $q_{s}=\left(V_{v a r} \uplus\right.$ $\left.V_{c o n}, L, E\right)$ consisting of a set of triple patterns $p\left(v_{1}, v_{2}\right)$. As usual, query answering amounts to graph pattern matching:

Definition 3. A match $q_{s}^{m}$ of a query $q_{s}$ on a graph $G$ is a homomorphic mapping $\mu_{s}$ from the variables of $q_{s}$ to vertices of $G$ such that the according substitution of variables in the graph pattern would yield a subgraph of $G$.

Query Translation. Translating $q_{u}$ means to find the queries $q_{s}$ representing possible interpretations of $q_{u}$. The focus lies on finding interpretations $q_{s}$ that have non empty results. In order for some graph pattern matches to exist for $q_{s}$, 

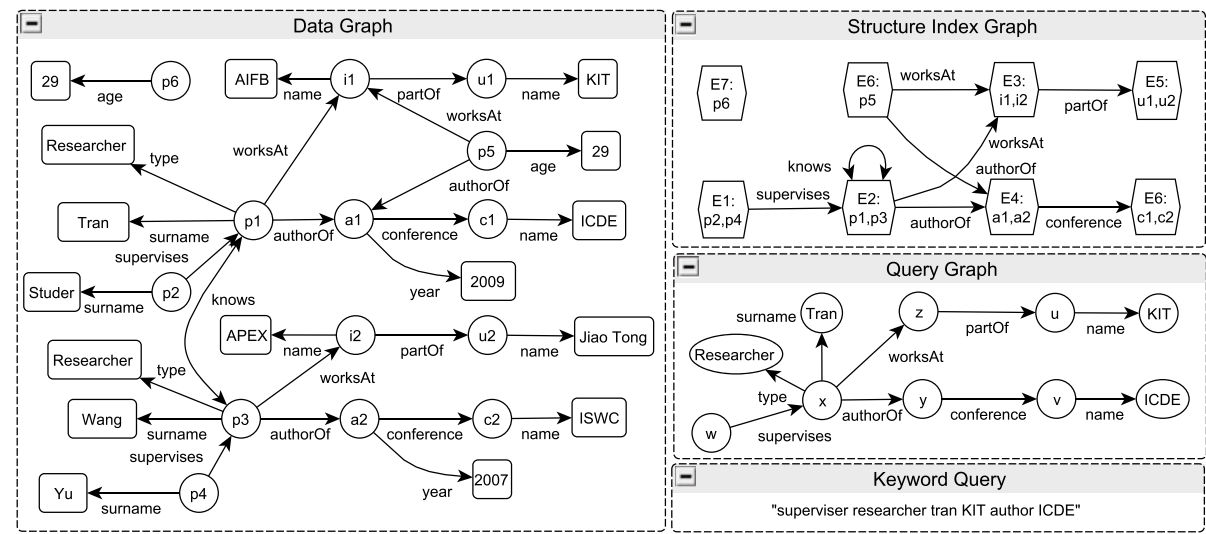

Fig. 1. a) A data graph, b) its structure index c) a query graph, d) a keyword query

predicates and constants of $q_{s}$ must correspond to some elements of $G$. Thus, we denote the space representing all possible interpretations of $q_{u}$ as $G_{q}$, a graph constructed using elements of $G$. An interpretation can be defined as a "keyword pattern" matching on $G_{q}$ :

Definition 4. An interpretation $q_{s}$ for $q_{u}$ is a subgraph of $G_{q}\left(V_{q}, L_{q}, E_{q}\right)$ connecting the keyword matching elements $n \in N_{k} \subseteq V_{q}$, where $n$ is obtained via a mapping $\mu_{u}$ from keywords of $q_{u}$ to elements in the set $V_{q} \uplus L_{q}$.

\section{Overview}

We propose a tight integration of query translation and query answering to obtain an incremental process such that intermediate results obtained via the first step can be reused in the second step. This is based on the observation that query translation is very similar to query answering. For query answering, triple patterns are matched against the data to obtain matching triples. The entire query graph is processed by performing joins on the matching triples along the query edges. During query translation, a similar matching is needed in order to obtain keyword matching elements. Exploration for matching subgraphs connecting these keyword elements is similar to join processing - with the difference that there are no query edges that can be used to guide this process.

The overall process is as follows: We firstly decompose the initial keyword query into segments, and map them to entities and relation labels of $G$. This step operates on two indexes built for $G$, one containing all relation labels $L_{R}$ of $G$, called relation label index, and another one containing all attribute edges $G\left(V, L_{A}, E\right)$ called entity index. The exploration for queries and search for candidate answers is combined in one step called join graph search. For this we employ a query search space $G_{q}$ constructed from a bisimulation-based structure index graph $G^{\sim}$, which represents the different edge-labeled structures that 
can be found in the data graph. Nodes of $G_{q}$ represent extensions which stand for entities in the data graph that are similar in structure. During join graph search, we explore $G_{q}$ for matching subgraphs from which candidate queries are derived. Because of the way the structure index graph is built from the data graph, these matching subgraphs are also candidate answers. That is, answers to the query are contained in the nodes (extensions) of the matching subgraphs and no other data has to be considered for query answering. Candidate answers in these extensions are refined in the subsequent query answering process. For this, the actual data graph edges have to be retrieved for elements in the extensions, using the relation index $G\left(V_{E}, L_{R}, E\right)$, and joined along the edges of the matching subgraphs in the last step.

Comparison to Related Work. The general top- $k$ subgraph exploration procedure proposed in [18] is adopted for join graph search. For brevity, we do not consider scoring models, while their use for top- $k$ and ranking as discussed in [18] is equally applicable to join graph search.

Different from work on direct keyword query answering 3129 , our approach aims to compute answers as well as queries that can be presented to the user. The exploration for subgraphs takes place on the structure index constructed from the data graph, instead of using the data graph itself.

So far, query translation has been performed independently from query answering 211/8]. Through the tight integration of these steps, we aim to minimize redundant processing by leveraging intermediate results. For this, we propose new algorithms for every major step of the integrated process: we decompose the keyword query into segments, from which the first initial result set of entities can be obtained. This is different to keyword mapping in previous approaches 2111839], where the aim is to find some matching elements (instead of entities). We propose the use of a structure index. As opposed to using the schema 21118, exploring the structure index results in a set of join graphs, which represent queries and at the same time, contain candidate answers that can be refined in the subsequent step. We propose a novel query answering procedure, which, as opposed to existing approaches [11915], accepts entities and the candidate answers contained in the join graphs as inputs.

\section{Keyword Mapping = Entity Search}

The first step to keyword search is keyword mapping, where the aim is to obtain a set of keyword matching elements. In direct keyword query answering, these elements are data elements, i.e. tuples or nodes of a data graph [39. For translation, keywords $k$ are mapped against the query search space $G_{q}\left(V_{q}, L_{q}, E_{q}\right)$ to obtain query elements (predicates and constants), i.e. $\mu: k \mapsto V_{q} \cup L_{q}$ 21118]. Since we are interested in queries with non-empty results, the computed query elements must match some elements of the data graph $G(V, L, E)$. In other words, query elements (and thus keywords) are assumed to be drawn from data graph element labels such that keyword mapping amounts to $\mu: k \mapsto V \cup L$. Thus, results of this 
mapping might comprise entities $v_{e} \in V_{E} \subset V$, data values $v_{d} \in V_{D} \subset V$ and edge labels $l \in L$. Keywords are matched against labels using standard IR-style keyword matching.

In the interest of computing not only queries but also answers, we propose an alternative mapping $\mu: k \mapsto G_{E} \cup L_{R}$, where $k$ might not be a keyword, but a keyword segment $k_{s} \subseteq q_{u}$. Such a segment refers to entity descriptions $G_{E}$ or relation labels $L_{R}$ and thus, are called entity keyword query and relation keyword query respectively:

Definition 5. A description of an entity $v_{e} \in V_{E}$ is a subgraph $G_{E}\left(V_{e}, L_{e}, E_{e}\right)$ of $G$ comprising all the edges $l\left(v_{e}, v_{d}\right) \in E$, where $v_{d} \in V_{D}$ (and thus $l \in L_{A}$ ). $A$ keyword $k$ denotes an entity $e$ if there is a mapping $\mu: k \mapsto G_{E}$, where $G_{E}$ are descriptions of entities $e \in E$.

An entity keyword query is a set of keywords $K_{e}$, where the intersection of the matching elements of all keywords is not empty, i.e. $\bigcap_{k \in K_{e}} G_{E_{k}} \neq \emptyset$ with $G_{E_{k}}$ being the matching descriptions and $e \in E$ being the matching elements for a keyword $k$.

$A$ keyword $k$ denotes a relation $r$ if there is a mapping $\mu: k \mapsto L_{R}$. Analogously, a relation keyword query is a set of keywords $K_{r}$, where the intersection of the matching relations of all keywords is not empty, i.e. $\bigcap_{k \in K_{r}} R_{k} \neq \emptyset$ with $R_{k}$ being the matching relation labels for keyword $k$.

In other words, we map keywords to entities and relation labels. For this, the keyword query $q=K$ is decomposed into a set of entity keyword queries $K_{E}=$ $K_{e_{1}}, \ldots, K_{e_{n}}$ and relation keyword queries $K_{R}=K_{r_{1}}, \ldots, K_{r_{n}}$. In particular, we are interested in partitions of the keywords, i.e. each keyword occurs in exactly one segment and there is no overlap between segments.

To create these partitions, we first generate segments of $K$ with non-empty results, i.e. matching segments. For this, the algorithm iterates through all subsets $S$ of $K$ in a bottom-up fashion, starting with single-element segments. The level corresponds to segment size such that at level 1, segments are of size 1 . At every level, a segment $s_{n} \in S$ is added to the set of matching segments $S^{m}$, if the following conditions are satisfied: its subsegment $s_{n-1}$ containing the keywords $\left\{k_{1}, \ldots, k_{n-1}\right\}$ has results (this is the case if $s_{n-1} \in S^{m}$ ); the additional keyword $k_{n} \in s_{n}$ has a result; and the intersection of these two sets of results is not empty. The final result, i.e. all valid combinations of keyword segments, is obtained via an algorithm to generate all partitions of the set $K$ [5], using the valid segments in $S^{m}$.

Example 1. For the keyword query in Fig. 1 d, we start with segments with length 1 , to see that all of them match some elements in $G$. At level 2, we find that only the combination researcher, tran is valid: $\mu($ researcher $) \cap \mu($ tran $)=$ $\{p 1\}$. Based on these valid segments, two partitions of the query are computed, i.e. $\{\{$ researcher,tran $\},\{K I T\},\{I C D E\},\{$ supervises $\},\{$ author $\}\}$ and $\{\{$ researcher $\},\{$ tran $\},\{K I T\},\{I C D E\},\{$ supervises $\},\{$ author $\}\}$. 


\section{Query Translation = Join Graph Search}

\subsection{Structure Index for Graph Structured Data}

Structure indexes have been widely used for semi-structured and XML data 4[13 16. A well-known concept for this is the dataguide [7, which basically is a structural description for rooted data graphs. Similar to this concept, a structure index has been proposed for general data graphs [17. Nodes of this structure index stand for groups of data elements that have equal structural "neighborhood", where equal structural neighborhood is defined by the wellknown notion of bisimulation. According to this notion, two graph nodes $v_{1}, v_{2}$ are bisimilar (written: $v_{1} \sim v_{2}$ ) if they cannot be distinguished by looking only at their outgoing or incoming "edge-labeled trees". Pairwise bisimilar nodes form an extension. Applying the bisimulation $\sim$ to the subgraph $G\left(V_{E}, L_{R}, E\right)$ of our data graph that contains relation edges only, results in a set of such extensions $\left\{[v]_{\sim}: v \in V_{E}\right\}$ with $[v]_{\sim}:=\left\{w \in V_{E}: v \sim w\right\}$. These extensions form a complete partition of the entity nodes $V_{E}$ of the data graph, i.e. form a family $\mathcal{P}_{\sim}$ of pairwise disjoint sets whose union is $V_{E}$.

Based on this notion of bisimulation, the structure index $G^{\sim}$ of $G\left(V_{E}, L_{R}, E\right)$ can be defined in terms of extensions and relations between them. In particular, extensions from the partition $\mathcal{P}_{\sim}$ form the vertices of $G^{\sim}$. An edge with label $l$ links two extensions $E_{1}, E_{2} \in \mathcal{P}_{\sim}$ of $G^{\sim}$ exactly if $G$ contains at least one $l_{r}$-edge linking an element in the extension $E_{1}$ to some element in the extension $E_{2}$.

Example 2. The data graph shown in Fig. 17 can be partitioned into 8 extensions, shown as nodes of the index graph in Fig. 1b. Nodes $p 1$ and $p 3$ are grouped into extension E2 as they are bisimilar, i.e. both have incoming supervise and knows edges and both have the same outgoing edgs paths knows, (worksAt,partOf) and (authorOf, conference).

Note that similar to the structure index, a schema also represents a structural description of the data. However, a structure index is a description of the structures actually exhibited by the data, constructed from the data. In particular, it has the following property [17:

Property 1. Whenever there is a match of a query graph $q$ on a data graph $G$ (homomorphism from $q$ into $G$ ) the query also matches on the index graph $G^{\sim}$ (homomorphism from $q$ into $G^{\sim}$ ). Moreover, nodes of the index graph matches will contain all data graph matches, i.e. the bindings to query variables.

\subsection{Construction of Query Search Space}

As opposed to direct keyword query answering, keyword query translation does not operate on the actual data $G(V, L, E)$, but on a query search space $G_{q}$. Essentially, this search space consists of two parts, (1) the keyword matching elements and (2) the structures that might connect these elements. Recall that in order for the computed queries $q_{s}$ to produce non empty results, all predicates 
and constants of $q_{s}$ must match elements of $G$. Hence, the query search space shall be constructed from elements in $G$. Recall that keyword matching elements comprise edge labels $l \in L_{R}$ and entities $e \in V_{E} \subseteq V$. Typically, the schema is used to represent the possible structures [2 1118]. Instead, we propose the use of a structure index to obtain the following query search space:

Definition 6. Given the set of keyword matching elements $n \in N^{k} \subseteq\left(L_{R} \uplus\right.$ $\left.V_{E}\right)$ and the data graph $G$, the query search space $G_{q}$ is defined as the index graph $G^{\sim}\left(V^{\sim}, L^{\sim}, E^{\sim}\right)$ of $G$, extended with a special type of edges of the form contains $\left([v]_{\sim}, n\right)$ iff $n \in V_{E}$ and $n$ is in the extension $[v]_{\sim} \in V^{\sim}$.

The query search space is thus the structure index graph, extended with keyword matching entity nodes. Since all different edge-labelled structures found in the data are represented in the structure index, all possible structures of queries with non empty results are completely captured by the proposed query search space. This result follows directly from Property 1 of the structure index:

Theorem 1. Whenever a query graph pattern $q$ matches a data graph $G$ (homomorphism from $q$ into $G$ ), there are some subgraphs of $G_{q}$ that match $q$ (homomorphism from $q$ into $G_{q}$ ).

Compared to the schema-based search space, the one proposed here is more appropriate for investigating possible translations. Since the schema does not necessarily represent actual structures in the data, many queries found through schema exploration might have empty results.

Example 3. Fig. 2a shows an example query search space, consisting of the structure index in Fig. 1 b extended with the keyword matching entities $p 1, u 1, c 1$.

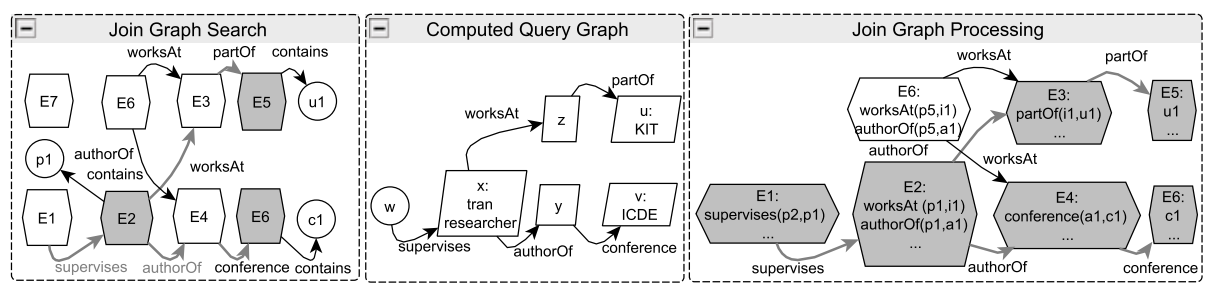

Fig. 2. a) Query space consisting of structure index, extended with keyword matching elements $p 1, u 1, c 1$, join graph found in this query space is highlighted, b) structured query corresponding to the join graph and c) the data retrieved and joined during join graph processing, join paths are highlighted.

\subsection{Search for Join Graphs}

During this step, we explore the query search space for subgraphs connecting the keyword elements. More precisely, these subgraphs called join graphs are formalized as follows: 
Definition 7. Let $G_{q}=\left(V_{q}, L_{q}, E_{q}\right)$ be the query search space, $K=$ $\left\{k_{1}, \ldots, k_{n}\right\}$ be the set of keyword segments and let $f: K \rightarrow V_{q} \cup L_{q}$ be a function that maps keyword segments to sets of corresponding graph elements. A K-matching join graph of $G_{q}$ is a graph $G_{q}^{m}=\left(V_{q}^{m}, L_{q}^{m}, E_{q}^{m}\right)$ with $V_{q}^{m} \subseteq V_{q}$ and $L_{q}^{m} \subseteq L_{q}$ such that

- for every $k \in K, f(k) \cap\left(V_{q}^{m} \cup L_{q}^{m}\right) \neq \emptyset$, i.e. $G_{q}^{m}$ contains at least one representative keyword matching element for every keyword from $K$, and

- $G_{q}^{m}$ is connected such that there exists a path from every graph element to every other graph element from $G_{q}^{m}$.

In our approach, we search for graphs, which contain the keyword matching edges $l \in L_{R}$, and additionally, can be used to join the keyword matching entities $e \in V_{E}$. Such join graphs are constructed using edges $e\left(\left[e_{1}\right]_{\sim},\left[e_{2}\right]_{\sim}\right) \in E^{\sim}$ of the structure index part of the query search space $G_{q}$. As several keyword matching elements might be contained in the same extension, we firstly compute the sets of extensions for every keyword segment $k$, which we use as starting points for the exploration.

We use the top- $k$ exploration algorithm as described in [18, to which we refer for an in-depth discussion of the algorithm. The algorithm is based on the concept of cursors, which represent an exploration path from a start element to some node in the structure index graph. Initially, each cursor is associated with a keyword segment and the corresponding extensions previously computed as starting elements. At each step, the cursor with the lowest cost (e.g. path length, but other metrics are also possible) is chosen for expansion. Also, the element at the end of the current cursor is examined to check whether it was explored by other cursors, so that all keyword segments are covered. If this is the case, the corresponding cursors are merged to form a subgraph. The exploration terminates when the top-k subgraphs are found or the maximum exploration distance is encountered for all cursors. Discovered subgraphs that are isomorphic are aggregated to obtain one single query for those representing the same "interpretations" of the keywords.

Example 4. This example is about finding the join graph $G_{q}^{m}=$ $\{$ supervises $(E 1, E 2)$, worksAt $(E 2, E 3), \operatorname{partOf}(E 3, E 5)$, authorOf $(E 2, E 4)$, conference $(E 4, E 6)\}$ as highlighted in Fig. 2. To obtain this, we iterate through $N^{k}=\{\{p 1\},\{u 1\},\{c 1\},\{$ supervise $\},\{$ author $\}\}$ to find the starting extensions $S^{k}=\{\{E 2\},\{E 5\},\{E 6\},\{$ supervise $\},\{$ author $\}\}$. At the beginning, all cursors created for these start elements have equal length. Thus, we might start with any element from $S^{k}$. For instance, we pop the cursor $c(E 2, E 2, \emptyset, 1)$ and add it to $E 2 . C_{E 2}$ to mark that $\mathrm{E} 2$ has been visited. Then, new cursors are created for neighbors \{supervises,authorOf,works At, contains $\}$ of E2. At the next iterations, remaining cursors of length 1 are taken from the queue and processed in the same manner. This proceeds until neighbor elements within distance of 4 have been explored for elements in $S^{k}$. Then, we find that E2 contains cursors to all other elements, i.e. E2. $(\{c(E 2, E 6$, authorOf, 4$)\},\{c(E 2$, supervises,supervises, 1$)\}$, 
$\{c(E 2$, authorOf,author $O f, 1)\},\{c(E 2, E 5$, works $A t, 4)\})$. Paths represented by these cursors are merged to obtain the join graph $G_{q}^{m}$. Further exploration does not yield any results not isomorphic to $G_{q}^{m}$.

Complexity. As shown in [18, the time needed for join graph search is bounded by $\left|G_{q}\right|^{k_{\max }}$ where $G_{q}$ is the query search space and $k_{\max }$ is the maximum path length of query atoms considered for query translation. When compared to direct keyword query answering based on exploring the data graph [312 9], this makes up a crucial difference in time complexity. In our approach, $\left|G_{q}\right|$ corresponds to the size of the structure index, which is bigger than the schema, but typically, is order of magnitudes smaller than the data graph [17.

\section{Query Answering = Join Graph Processing}

Instead of retrieving triples and joining them along the query, as done in standard query processing, we propose query answering to be tightly coupled with query translation, such that the computed entities and join graphs corresponding to the user queries can be leveraged.

Recall that the join graphs $G_{q}^{m}$ computed during translation are subgraphs of the structure index. Every node of such a graph represents an extension, i.e. a set of entities. Due to Property 1, we can infer that the structure index enables us performing query translation and answering in an incremental way:

Theorem 2. Extensions of a join graph $G_{q}^{m}$, from which the query $q$ has been derived, contain all the bindings to variables of $q$.

Proof 1. Query translation is performed based on the isomorphism from $G_{q}^{m}$ into $q$. Thus, $G_{q}^{m}$ is a match of $q$, i.e. homomorphism from $q$ into $G_{q}$. Since the structure index $G^{\sim}$ part of $G_{q}$ is the one used for the exploration, $G_{q}^{m}$ is in fact a homomorphism from $q$ into $G^{\sim}$, i.e. it is an index graph match. By Property $1, G_{q}^{m}$ contains all the bindings to $q$.

Intuitively speaking, not only queries but also candidate answers were computed during translation. These answers are contained in $G_{q}^{m}$. Thus, it suffices to focus on elements in the extensions of $G_{q}^{m}$. More precisely, we can focus on the keyword matching entities in these extensions.

The query answering algorithm leveraging these intermediate results is shown in Alg. 1. Given the join graph $G_{q}^{m}$ including the keyword matching elements $e \in N^{k}$ contained in some nodes $[e]_{\sim}$ of $G_{q}^{m}$ (i.e. $e$ is connected with $[e]_{\sim}$ via $\left.\operatorname{contains}\left([e]_{\sim}, e\right)\right)$, it computes all matches of this pattern on the data graph $G=(V, L, E)$. These matches are stored as tuples in the result table $R$. Just like standard query processing on graph structured data 11915, edges of the query pattern $G_{q}^{m}$ are processed by retrieving triples $E\left(l_{q}^{m}\right)$ from the data graph matching the edge $l_{q}^{m}\left(\left[e_{1}\right]_{\sim},\left[e_{2}\right]_{\sim}\right)$. However, if given results exist, i.e. there are keyword matching entities contained in $\left[e_{1}\right]_{\sim}$ or $\left[e_{2}\right]_{\sim}$ such that $\left[e_{1}\right]_{\sim} \cdot N^{k}$ or $\left[e_{2}\right]_{\sim} \cdot N^{k}$ is not empty, they are used for further processing. Otherwise, triples 


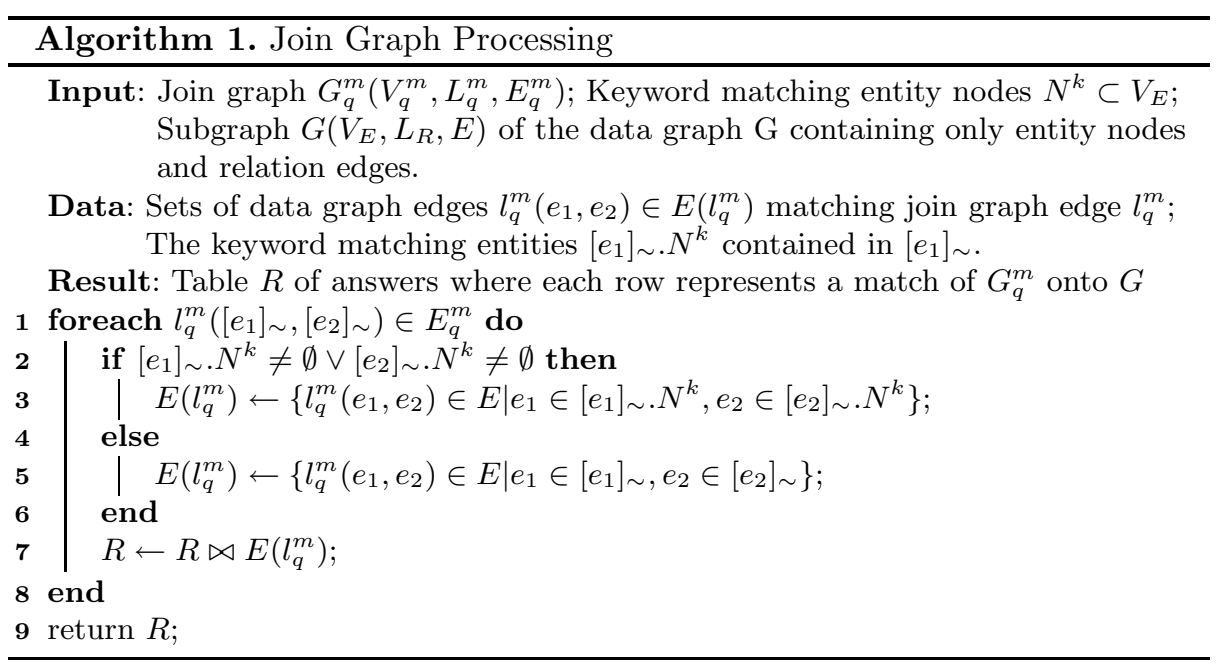

$l_{q}^{m}\left(e_{1}, e_{2}\right)$ are retrieved from $G$. Due to the use of the structure index, only triples with $e_{1} \in\left[e_{1}\right]_{\sim}$ and $e_{2} \in\left[e_{2}\right]_{\sim}$ have to be retrieved. This number of triples might be considerably lower than all the triples with label $l_{q}^{m}$.

Example 5. For processing the translated query $q$ shown in Fig. 2b, triples are retrieved and joined along the edges $L_{q}^{m}=$ \{supervise,worksAt, authorOf,partOf,conference\} of the corresponding join graph $g$ shown in Fig. 2a. If we start with supervise $(E 1, E 2)$ for instance, we found that $E 2 . N^{k}=p 1$. Thus, $E\left(l_{q}^{m}\right)=\operatorname{supervises}(p 2, p 1)$, i.e. only one edge with $p 1$ is retrieved. In this example, either $\left[e_{1}\right]_{\sim} \cdot N^{k}$ or $\left[e_{2}\right]_{\sim} \cdot N^{k}$ is always $\neq \emptyset$. Join graph processing for this example and the result $R=\{\operatorname{supervises}(p 2, p 1)$, works At $(p 1, i 1)$, partOf $(i 1, u 1)$, conference $(a 1, c 1)\}$ is illustrated in Fig. 22.

Complexity. As shown in [17], for a join graph with $\left|E_{q}^{m}\right|$ edges, the time and space for computing the answer table $R$ is bounded by $O\left(\operatorname{edgemax}^{\left|E_{q}^{m}\right|}\right)$ where edgemax is $\left|E\left(l_{q}^{m}\right)\right|$, with $l_{q}^{m}$ being the label instantiated by the largest number of edges. This complexity result holds also for existing query answering approaches, where $\left|E\left(l_{q}^{m}\right)\right|$ is derived from size of the tables built for edge labels $l \in L$. The crucial difference lies in this factor. In our approach, $\left|E\left(l_{q}^{m}\left(\left[e_{1}\right]_{\sim},\left[e_{2}\right]_{\sim}\right)\right)\right|$ amounts to $\max \left\{\left|\left[e_{1}\right]_{\sim} \cdot N^{k}\right|,\left|\left[e_{2}\right]_{\sim} \cdot N^{k}\right|\right\}$, i.e. the number of entities computed during query mapping, which are contained in the join graph nodes $\left[e_{1}\right]_{\sim}$ and $\left[e_{2}\right]_{\sim}$. If no entities have been obtained for either one of these nodes, $\left|E\left(l_{q}^{m}\left(\left[e_{1}\right]_{\sim},\left[e_{2}\right]_{\sim}\right)\right)\right|$ amounts to $\max \left\{\left|\left[e_{2}\right]_{\sim}\right|,\left|\left[e_{2}\right]_{\sim}\right|\right\}$, i.e. the size of the join graph nodes.

\section{Evaluation}

Through the experiments, we aim to compare performance of our approach against the state of the art. 
Systems. For implementing the underlying indexes, we use a standard technique for indexing graph structured data. In particular, we use inverted indexes, as elaborated in [6]. We use an IR engine 1 for managing these indexes. This engine is also leveraged for mapping keywords to relation edge labels and entities (keyword mapping), as well as for the retrieval of relations (join graph processing). We compare our system against the keyword translation approach elaborated in [18. It has been shown that the time for query translation achieved through [18, plus the time for query answering is lower than the time for the state of the art system based on direct keyword query answering [3[12]9. For a detailed comparison with the state of the art approaches on direct keyword query answering, we refer to 18 .

We have implemented this translation approach (QT) on top of a system for query answering (QA) based on vertical partitioning [1] and compared it against the implementation of our integrated approach (IQTQA). All implementations rely on the same engine for indexing and retrieval.

Datasets. The following datasets are used for the benchmark: $D B L P$ captures bibliographic information about the field of Computer Science. It has been a standard dataset for evaluating keyword search [918. LUBM is the Lehigh University benchmark, a synthetic dataset used extensively in the Semantic Web community for evaluating knowledge base systems and RDF stores 8 . We used the data generator proposed for the LUBM benchmark to create datasets for 1 , 5,10 and 50 imaginary universities.

Table 1. Statistics for the data graphs and indexes

\begin{tabular}{|l|l|l|l|l|l|l|}
\cline { 2 - 7 } \multicolumn{1}{c|}{} & Data [\#Edges $]$ & Data [MB $]$ & $\begin{array}{l}\text { EntityIdx } \\
{[\mathrm{MB}]}\end{array}$ & $\begin{array}{l}\text { RelIdx } \\
{[\mathrm{MB}]}\end{array}$ & $\begin{array}{l}\text { StrucIdx } \\
{[\mathrm{KB}]}\end{array}$ & $\begin{array}{l}\text { Schema } \\
{[\mathrm{KB}]}\end{array}$ \\
\hline DBLP & $12,920,826$ & 2,084 & 2210 & 2311 & 132 & 28 \\
\hline LUBM1 & 100,577 & 17 & 20 & 16 & 92 & 24 \\
\hline LUBM50 & $6,654,596$ & 1,132 & 1391 & 1,037 & 82 & 24 \\
\hline
\end{tabular}

For these datasets, the size and number of edges are shown in Table 1. Also, the size of the associated entity index, relation index, structure index and schema is provided. One can see that the structure index is consistently bigger than the schema, but is of magnitudes smaller than the data graph. Further, its size is not dependent on the size of the data graph, but the structures contained in it.

Queries. In order to study the behavior of the proposed algorithms in a principled way, test queries are generated by random sampling from the data. Keyword queries are derived from generated structured queries.

Setting. We carried out the experiments on a Linux machine with two Intel Xeon Dual Core $2.33 \mathrm{GHz}$ processors and $48 \mathrm{~GB}$ of main memory, of which 2GB were allocated to the Java VM. All data and indexes were stored on a RAID

${ }^{1}$ http://lucene.apache.org/ 
array. All times presented are the average of 10 runs of 25 generated queries for each dataset. Between queries, we explicitly clear the operating system cache and internal caches.

Total Processing Time. We have measured the average processing time needed for QTQA and our approach IQTQA. For QTQA, we take the translation time + the query answering time for one query, the time needed for answering the first query output by query translation. For IQTQA, we consider query answering time needed for processing the first join graph, as well as the time needed for processing all join graphs output by query translation. As shown in Fig. [3], query translation constitutes the greater share. Especially in our approach, query translation makes up for more than $90 \%$ of the total processing time. This is because we have chosen a generous value for $k_{\max }$, resulting in a relatively large neighborhood to be explored and a large number of candidate interpretations to be computed. Consistently for all datasets, query translation using QTQA is faster than our approach IQTQA. The difference between these two approaches is greater for the LUBM datasets, where QTQA is up to 20 percent faster w.r.t query translation. This advantage of QTQA over IQTQA is however outweighed by the worse performance in query answering. While query answering takes almost half of the total time for QTQA, it makes up only a small fraction of the total time for IQTQA. This holds for all datasets. This even holds when not only the first join graph, but all join graphs are processed for IQTQA, i.e. query translation + computing answers for all computed queries using IQTQA is faster than query translation + computing answers for only one query using QTQA, w.r.t all datasets. It seems that most of the work has been done already during query translation such that the computation of answers for all computed queries consume only a small fraction of additional time.

Effect of Data Size. We have measured total time for LUBM of different data sizes. As illustrated in Fig. 3a a query translation time in both QTQA and IQTQA increases linearly with the size of the data. Further decomposition of this time into its two main components tells that this increase is mainly due to query mapping, while the time for join graph search remains relatively constant. This is consistent with our complexity analysis. The time for join graph search depends on the structure index. Since the size of the structure indexes (and schemas) is relatively constant in our experiments, this part of query translation is not affected by the changes in data size. However, query mapping time is partly determined by the number of mappings that can be retrieved from the data. Larger data sizes lead to larger number of mappings, which in turn, result in higher mapping time. Also query answering time increases linearly with the size of the data, for both QTQA and IQTQA. This is not surprising, as this time component is determined by the number of triples that can be retrieved for a query atom. Larger data size results in larger number of matching triples.

Effect of Keyword Query Complexity. We have run experiments with keyword queries of different complexity, as measured by the number of keywords. The query translation time for QTQA and IQTQA is illustrated in Fig. 3r for 


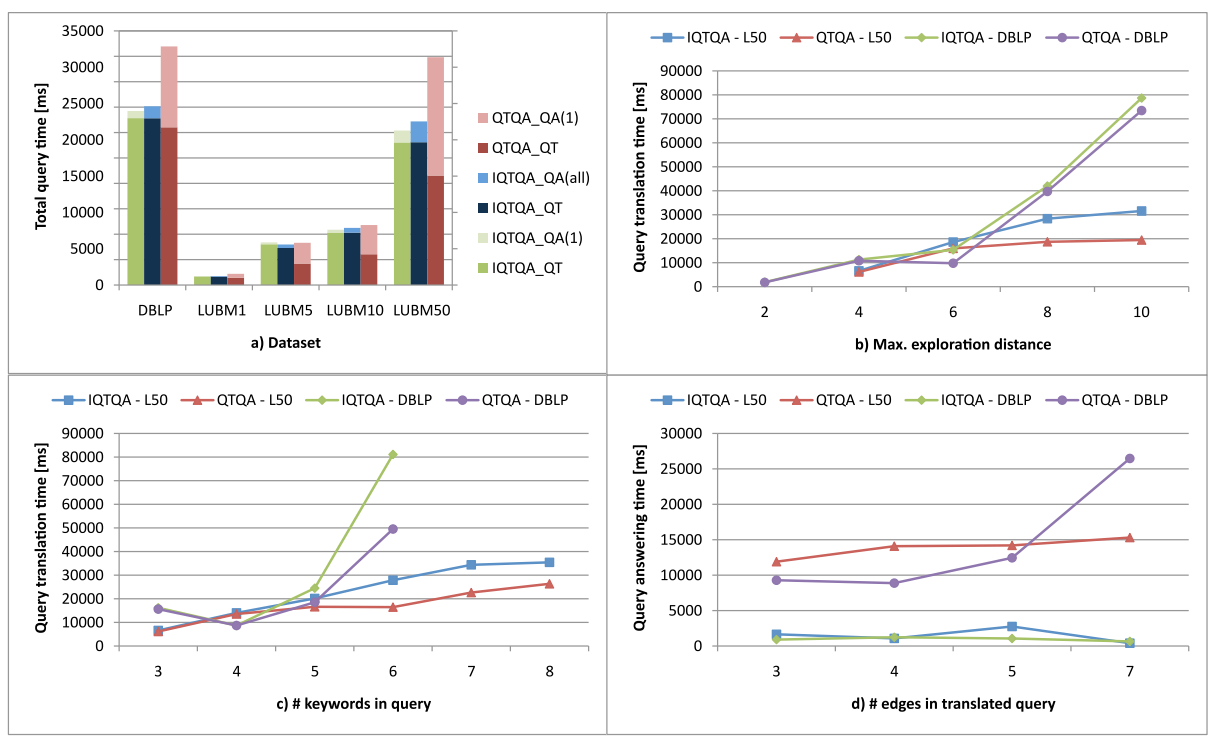

Fig. 3. Evaluation results

DBLP and LUBM50. Query translation time increases with the number of keywords. This is because the number of keywords has an influence on the complexity of both keyword mapping and join graph search. When the number of keywords increase, a larger number of mappings needs to be retrieved, a larger number of segments require to be tested and potentially, a larger number of starting elements have to be considered during join graph search. Compared with QTQA, the increase exhibited by IQTQA is slightly stronger. This can be explained by the larger search space employed by IQTQA, i.e. the structure index is bigger than the schema. The negative effect of a larger number of starting elements exacerbate, when the search space is larger in size.

Effect of Maximum Neighborhood Distance. This problem is more evident when looking at the maximum neighborhood size $k_{\max }$ used for join graph search. We illustrate the effect of this factor in Fig. 3 b. The increase of query translation time with greater $k_{\max }$ is consistently stronger for IQTQA, especially w.r.t LUBM50. A greater $k_{\max }$ means simply means a greater portion of the search space will be searched, thus resulting in higher translation time.

Effect of Structured Query Complexity. The negative effect due to the larger search space is overcompensated by the gain in query answering performance. In Fig. 3d, we illustrate the relation between query answering time and query complexity, where complexity is represented by the number of query edges. While time for QTQA increases linearly, time for IQTQA remains relatively constant. Here the result for QTQA is better than worst case performance because in many instances, retrieved data comes in sorted order, thus enabling fast joins. 
Query times for IQTQA do not increase with the number of query edges because for most cases, intermediate results from translation have been leveraged such that only a small number of data elements are actually involved in the join.

\section{Conclusion}

We have proposed an approach for keyword search, which combines query translation with structured query answering to obtain an integrated process. Results produced during translation are reused to improve the efficiency of subsequent query answering. For this, we break down the overall process into two similar pattern matching problems: one is to match the keywords against a query search space to obtain a query, and the other is to match the query against the data. By constructing the query search space based on the data, i.e. the entities and the structures connecting them as represented by a structure index, the result of the first matching can greatly improve the efficiency of the second matching. We propose algorithms to perform these two matching tasks in an efficient and incremental way. We have established complexity bounds for these algorithms and compare these theoretical results with related work. In the benchmark against the state of the art, we show that our approach outperforms the state of the art.

Acknowledgements. Research reported in this paper was supported by the German Federal Ministry of Education and Research (BMBF) under the iGreen project (grant 01IA08005K).

\section{References}

1. Abadi, D.J., Marcus, A., Madden, S., Hollenbach, K.J.: Scalable Semantic Web Data Management Using Vertical Partitioning. In: VLDB, pp. 411-422 (2007)

2. Agrawal, S., Chaudhuri, S., Das, G.: DBXplorer: enabling keyword search over relational databases. In: SIGMOD Conference, p. 627 (2002)

3. Bhalotia, G., Hulgeri, A., Nakhe, C., Chakrabarti, S., Sudarshan, S.: Keyword searching and browsing in databases using banks. In: ICDE, pp. 431-440 (2002)

4. Buneman, P., Davidson, S., Fernandez, M., Suciu, D.: Adding Structure to Unstructured Data. In: Afrati, F.N., Kolaitis, P.G. (eds.) ICDT 1997. LNCS, vol. 1186, pp. 336-350. Springer, Heidelberg (1997)

5. Djokic, B., Miyakawa, M., Sekiguchi, S., Semba, I., Stojmenovic, I.: A Fast Iterative Algorithm for Generating Set Partitions. Comput. J. 32(3), 281-282 (1989)

6. Dong, X., Halevy, A.Y.: Indexing dataspaces. In: SIGMOD Conference, pp. 43-54 (2007)

7. Goldman, R., Widom, J.: DataGuides: Enabling Query Formulation and Optimization in Semistructured Databases. In: VLDB, pp. 436-445 (1997)

8. Guo, Y., Pan, Z., Heflin, J.: LUBM: A benchmark for OWL knowledge base systems. J. Web Sem. 3(2-3), 158-182 (2005)

9. He, H., Wang, H., Yang, J., Yu, P.S.: Blinks: ranked keyword searches on graphs. In: SIGMOD Conference, pp. 305-316 (2007)

10. Hristidis, V., Gravano, L., Papakonstantinou, Y.: Efficient ir-style keyword search over relational databases. In: VLDB, pp. 850-861 (2003) 
11. Hristidis, V., Papakonstantinou, Y.: DISCOVER: Keyword Search in Relational Databases. In: VLDB, pp. 670-681 (2002)

12. Kacholia, V., Pandit, S., Chakrabarti, S., Sudarshan, S., Desai, R., Karambelkar, H.: Bidirectional Expansion For Keyword Search on Graph Databases. In: VLDB, pp. 505-516 (2005)

13. Kaushik, R., Bohannon, P., Naughton, J.F., Korth, H.F.: Covering indexes for branching path queries. In: SIGMOD Conference, pp. 133-144 (2002)

14. Liu, F., Yu, C.T., Meng, W., Chowdhury, A.: Effective keyword search in relational databases. In: SIGMOD Conference, pp. 563-574 (2006)

15. Neumann, T., Weikum, G.: Rdf-3x: A risc-style engine for RDF. PVLDB 1(1), 647-659 (2008)

16. Qun, C., Lim, A., Ong, K.W.: D(k)-Index: An Adaptive Structural Summary for Graph-Structured Data. In: SIGMOD Conference, pp. 134-144 (2003)

17. Tran, T., Ladwig, G., Rudolph, S.: Efficient RDF Data Management Using Structure-based Partitioning and Structure-aware Query Processing. Technical report, http://www .aifb.uni-karlsruhe.de/WBS/dtr/papers/strucidxTR.pdf

18. Tran, T., Wang, H., Rudolph, S., Cimiano, P.: Top-k Exploration of Query Candidates for Efficient Keyword Search on Graph-Shaped (RDF) Data. In: ICDE, pp. 405-416 (2009)

19. Weiss, C., Karras, P., Bernstein, A.: Hexastore: sextuple indexing for semantic web data management. PVLDB 1(1), 1008-1019 (2008) 